\title{
Automatic Diagnosis of Lumbar Disc Herniation with Shape and Appearance Features from MRI
}

\author{
Raja' S. Alomari ${ }^{a}$, Jason J. Corso ${ }^{a}$, Vipin Chaudhary ${ }^{a}$, Gurmeet Dhillon $^{b}$ \\ \{ralomari, jcorso, vipin\}@cse.buffalo.edu \{gdhillon\}@proscan.com \\ ${ }^{a}$ University at Buffalo, SUNY, Buffalo, NY 14260 \\ ${ }^{b}$ Proscan Imaging of Buffalo, Williamsville, NY 14221
}

\begin{abstract}
Intervertebral disc herniation is a major reason for lower back pain (LBP), which is the second most common neurological ailment in the United States. Automation of herniated disc diagnosis reduces the large burden on radiologists who have to diagnose hundreds of cases each day using clinical MRI. We present a method for automatic diagnosis of lumbar disc herniation using appearance and shape features. We jointly use the intensity signal for modeling the appearance of herniated disc and the active shape model for modeling the shape of herniated disc. We utilize a Gibbs distribution for classification of discs using appearance and shape features. We use 33 clinical MRI cases of the lumbar area for training and testing both appearance and shape models. We achieve over $91 \%$ accuracy in detection of herniation in a cross-validation experiment with specificity of $91 \%$ and sensitivity of $94 \%$.
\end{abstract}

Keywords: Lumbar Spine, Herniation, Computer Aided Diagnosis, MRI.

\section{INTRODUCTION}

Lower back pain (LBP) is the second most common neurological ailment in the United States after the headache. ${ }^{1}$ It is reported that Americans spend at least $\$ 50$ billions each year on medical diagnosis and rehabilitations related to lower back pain. ${ }^{1}$ Intervertebral disc degeneration (e.g., herniation) in the lumbar area is one of the most common diseases that cause LBP and sciatica, a common term for pain in legs consequent to irritation of the sciatic nerve. ${ }^{2,3}$ Furthermore, Over $90 \%$ of surgical spine procedures are performed because of consequences of the degenerative process. ${ }^{4}$

Diagnosis of most backbone abnormalities (including disc degeneration and herniation) are performed, in clinics, by radiologists based on studying clinical MRI. Clinical MRI usually comprises sagittal T1- and T2weighted (manually) co-registered protocols beside others to help the radiologists in decision-making. For diagnosis of herniation, they might also use axial views for confirmation of their decision (especially for quantification of the herniation). Because disc signal intensity in T2-weighted MRI is the most sensitive sign for intervertebral disc degeneration, radiologists tend to use T2-weighted for diagnosis of degenerative disc related abnormalities in most cases. ${ }^{4}$

Building computer aided diagnosis (CAD) systems have been attracting many researchers and clinicians for many decades. Many of them have been built such as 1) CAD using CT for detection (or diagnosis) of colonic polyp $^{5,6}$ and lung nodules from $\mathrm{CT},{ }^{7}$ 2) CAD using mammography for detection of breast cancer, $\left.{ }^{8} 3\right) \mathrm{CAD}$ using MRI for detection of breast ${ }^{9}$ and prostate ${ }^{10}$ cancer. More recently, major attention has been given to incorporation of these CAD systems within the work flow of clinical diagnosis as this has been a a barrier for CAD use in clinics. ${ }^{11,12}$ We work on building a full CAD system that includes automation of most lumbar area abnormalities diagnosis and, simultaneously, incorporating this CAD systems as plug ins into the work flow of our collaborating radiologist in a testing environment. ${ }^{13-16}$

In this paper, we present a method that automates the diagnosis of disc herniation. Our method incorporates shape and intensity features to model the herniated disc and flag it. We use both T1- and T2-weighted coregistered sagittal views for building a 2 D feature image I. We then train an active shape model (ASM) ${ }^{17}$ for modeling the disc shape. We then extract a set of empirically-sound features to diagnose herniated discs. We also model the appearance of the disc based on the normalized intensity signal similar to our previous work. ${ }^{13,14}$ Then we build a probabilistic classifier by introducing the random variable $n$ and solving: 


$$
n^{*}=\arg \max _{n} P(n \mid \mathrm{S}, \mathrm{A})
$$

where $n$ is a binary random variable stating whether it is a herniated or a normal disc, $\mathrm{S}$ represents the shape features extracted from the lumbar disc ASM, and A represents the appearance features.

The remainder of this paper is organized as follows: Background and related work is discussed in section 2. Then we provide a description of our dataset in section 3 . We then present our method in section 4 , our experimental results in section 5, and conclude in section 6 .

\section{BACKGROUND AND RELATED WORK}

Intervertebral disc herniation is a medical condition affecting the spine in which a tear in the outer ring, annulus fibrosus, allows the inner soft jelly-like substance, nucleus pulposus, to bulge out. Fig. 2 shows an axial view model for the anatomy of the disc and a description of the herniation. ${ }^{18}$ In MRI data, herniation can be detected in the sagittal view and it typically appears as shown in Fig. 1.

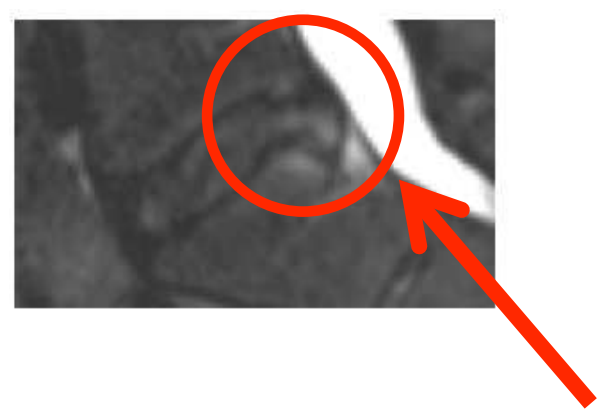

Figure 1. A $7 \mathrm{~mm}$ herniated disc.

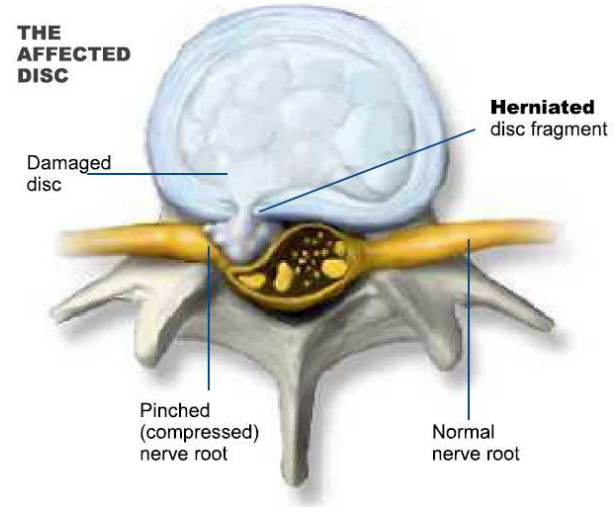

Figure 2. Herniated disc model ${ }^{18}$

Many researchers have proposed methods for the diagnosis of certain vertebral column abnormalities. Bounds et al. ${ }^{19}$ utilized a neural network for diagnosis of back pain and sciatica. Sciatica might be caused by lumbar disc herniation as well as many other reasons. They have three groups of doctors to perform diagnosis as their validation mechanism. They achieved better accuracy than the doctors in the diagnosis. However, the lack of data prohibited them from full validation of their system. Similarly, Vaughn ${ }^{20}$ conducted a research study on using neural network for assisting orthopedic surgeons in the diagnosis of lower back pain. They classified LBP into three broad clinical categories. They used 25 features to train the Neural Network (NN) including symptoms clinical assessment results. The NN achieved $99 \%$ of training accuracy and $78.5 \%$ of testing accuracy. This clearly shows training data overfitting.

Tsai et al. ${ }^{21}$ used geometrical features (shape, size and location) to diagnose herniation from 3D MRI and CT axial (transverse sections) volumes of the discs. In contrast, we do not presume the availability of the full volume axial view as it is not a clinical standard. We also jointly make use of appearance and shape information."

Roberts et al. ${ }^{22}$ used ASM to detect and quantify vertebral fracture from x-ray radiographs for the lumbar and thoracic area (L4 up to T7) using extracted shape and appearance features for performing quantitative fracture classification. Because of differences in vertebrae, they trained a shape model for each of three classes: upper thoracic (T7-T9), lower thoracic (T10-T12), and lumbar (L1-L4). They presented a comparison study between appearance and shape effect on classification in each vertebral group.

In this paper, we diagnose disc herniation from sagittal views which is different from Tsai et al. ${ }^{21}$ However, we utilize some features of the shape model that Roberts et al. ${ }^{22}$ used in detection and quantification of fractures. Though we build our model on the discs and not on the vertebrae. Finally, our data is clinical MRI and not x-rays. 


\section{DATASET AND PREPARATION}

We use a clinical dataset where herniation is the major abnormality obtained by our collaborating clinical research group. For this paper, we use thirty three cases where each case contains at least one herniated disc in the lumbar area. However, many cases have only one herniated disc and the rest are normal. Each case has two sagittal co-registered MRI protocols: T1- and T2-weighted. This registration is performed manually by the MRI technician as this is the clinical standard.

These cases are acquired by Philips MRI 1.5 Tesla scanners that are used for clinical diagnosis of various vertebral column abnormalities. To reduce the effect of magnetic field inhomogeneities in MRI, radiologists use cerebrospinal fluid $(\mathrm{CSF})^{23}$ or the spinal signal ${ }^{24}$ as a standard reference for disc intensity levels. We normalize the intensity using the spinal signal to avoid related issues of magnetic field inhomogeneities.

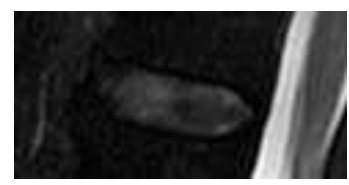

(a) Normal Disc at L1-L2 level.

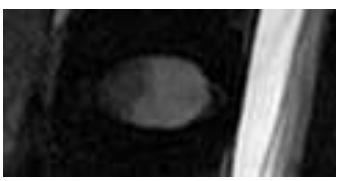

(b) Normal Disc at L2-L3 level.

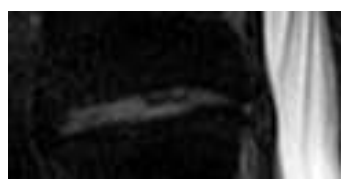

(c) Herniated Disc at L4L5 level.

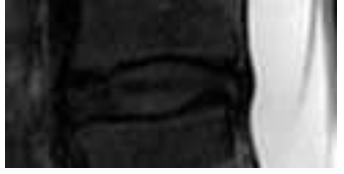

(d) Herniated Disc at L4L5 level.

Figure 3. Variations in normal and herniated disc shapes.

To localize the discs, we use our previous labeling method in Corso et al. ${ }^{13}$ which results in a point inside each of the lumbar discs. Then we obtain a fixed window of size $60 \times 120$ pixels centered at the labeling point as shown in Fig. 3. We then manually check that the window size is suitable to enclose the whole disc with a portion from the spine for the whole dataset for training purposes.

\section{METHOD}

Shape is a key player in detection of herniation due to the major shape-change caused by herniation, as shown in Fig. 3(c). On the other hand, intensity signal levels for herniated discs are usually lower than normal discs because when the inner pulposus leaks out (herniates), the water contents of the disc spreads over larger area as shown in Fig. 3(d). However, lower intensity levels of a disc might indicate other abnormalities such as desiccation. ${ }^{14}$ Thus, we jointly use both the shape and the appearance features for maximum effectiveness.

\subsection{Shape (S)}

Sagittal views of lumbar intervertebral discs generally have elliptical shapes as shown in Fig. 3, but the shape varies depending on patient's age, height, normality condition, and many other reasons. The variations in disc shapes affect their size, major and minor diameters as shown in Fig. 3(a) (b). Herniated discs might, sometimes, change the shape of the exterior end of the disc as shown in Fig. 3(c).

However, given the roughly elliptical disc shape, even under

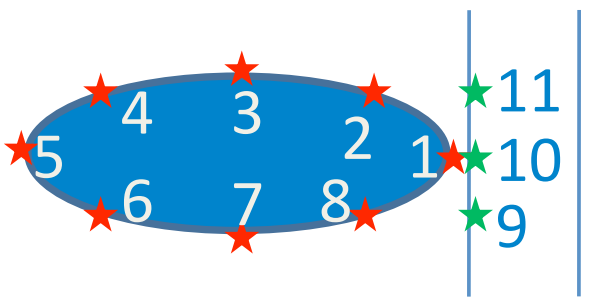

Figure 4. Point Distribution of the training data. herniation, we assume the underlying manifold of variations is roughly linear. We hence use an active shape model $^{25}$ for learning the shape variations of the lumbar disc. We use 11 landmark points $\mathrm{S}=\left\{s_{i}: i=1, \ldots, 11\right\}$ to represent each shape which includes the disc boundary and the spine portion connected to the disc. Fig. 4 shows the distribution of these points on an illustrative model.

To increase the effectiveness of using the ASM, rather than working with the original T1 and T2 images, we define a range filter, $\mathrm{R}$, to compute a feature image, $\mathrm{I}$ :

$$
\mathrm{I}=\mathrm{R}(\mathrm{T} 1+\mathrm{T} 2)
$$


where T1 and T2 are the normalized T1- and T2-weighted MRI image for the same case. These two images are physically co-registered during acquisition of MRI. $\mathrm{R}$ is the range filter operator where intensity levels in each $3 \mathrm{x} 3$ window are replaced by the range value (maximum - minimum) in that window. This operator $\mathrm{R}$ has high values in abrupt-change regions and small values in smooth regions which results in a better feature image than the original T2- or T1-weighted as shown in Fig. 5 compared to Fig. 3. For the ASM, this improves both the convergence speed and accuracy to localize the model landmark points during inference.

We prepare the training data for the ASM manually by selecting the set of points on the feature image I as shown in Fig. 5. The ASM learns the distribution of shapes by initially calculating the mean shape $\bar{x}=\frac{1}{N} \sum_{1}^{N} x$ where $N$ is the size of the training data. Then each disc shape $x_{i}$, where $i \in\{1, \ldots, N\}$ and $N$ is the size of the training set, is recursively aligned to the mean shape $\bar{x}$ using generalized Procrustes analysis to remove translational, rotational, and isotropic scaling from the shape. ${ }^{17}$

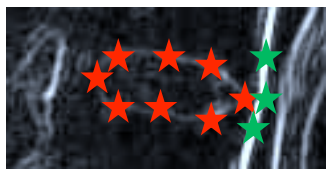

(a) Normal Disc at L2-L3 level.

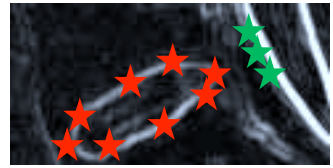

(b) Normal Disc at L5-S level.

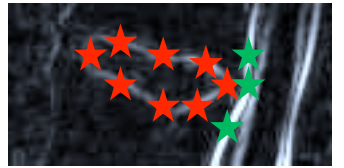

(c) Normal Disc at T12L1 level.

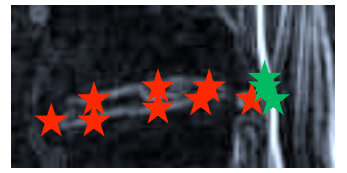

(d) Herniated Disc at L4L5 level.

Figure 5. Feature image with shape points distribution.

Then, we model the remaining variance around the mean shape with principal components analysis (PCA) to extract the eigenvectors of the covariance matrix associated with $98 \%$ of the remaining point position variance according to the standard method for deriving the ASM's linear shape representation. ${ }^{17}$

\subsection{Appearance (A)}

Intensity levels of herniated discs are usually less than normal discs because the nucleus pulposus spreads over a larger area as shown in Fig. 3(d) and thus the signal intensity becomes lower. We model the appearance A of herniated discs based on a pixel neighborhood $\sigma_{\mathrm{I}_{d}}$ surrounding the disc point $d$, which is provided during the initial localization step. Herniated disc intensities have a general Gaussian shape with lower mean value $\mu_{\text {I }}$ than the normal disc intensities distribution. ${ }^{13,14}$ Fig. 6 shows the intensity distribution of a set of normal discs in T2-weighted MRI. We fit a Gaussian model for this distribution as illustrated by next section. We also assume a Gaussian fit for herniated disc intensities due to the insufficient amount of herniated discs to establish a well-defined distribution, though this assumption is supported by our empirical results.

\subsection{Classification and Testing}

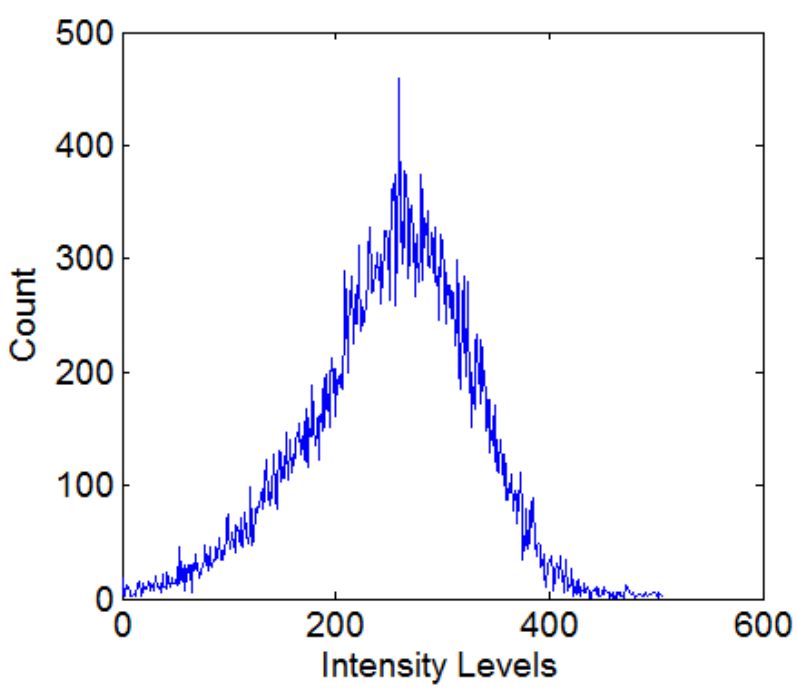

Figure 6. Intensity distribution for normal discs.

Our classifier models both the shape $\mathrm{S}$ and the appearance $\mathrm{A}$ of the disc. The shape features are extracted from the point distribution of the ASM while the appearance features are extracted from a pixel neighborhood $\sigma_{I_{d}}$ surrounding the point $d$ inside the disc. We capture herniation $n$ with a Gibbs model:

$$
P(n \mid \mathrm{S}, \mathrm{A})=\frac{1}{Z[n]} \exp ^{-\left[\beta_{1} * U_{\mathrm{A}}(\mathrm{A})+\beta_{2} * U_{\mathrm{S}}(\mathrm{S})\right]}
$$


where $n$ is a binary random variable for the herniated disc, S represents the shape features defined by the ASM, A represents the appearance features defined by a neighborhood of pixels $\sigma_{\mathrm{I}_{d}}$ around the point $d$ inside the disc, $Z[n]$ is the normalization factor of the Gibbs distribution, $\beta_{1}$ and $\beta_{2}$ are tuning parameters, $U_{\mathrm{A}}$ and $U_{\mathrm{S}}$ are the appearance and shape potentials, respectively.

The appearance potential $U_{\mathrm{A}}$ models the intensity levels of the herniated disc as a Gaussian. ${ }^{13,14}$ We take the negative log and the potential is then given by:

$$
U_{\mathrm{A}}\left(\mathrm{A} ; \sigma_{\mathrm{I}_{d}}\right)=\frac{\sum_{j \in \sigma_{\mathrm{I}_{d}}}\left(\mathrm{I}(j)-\mu_{\mathrm{I}}\right)^{2}}{2 \sigma_{\mathrm{I}}^{2}}
$$

where $d$ is the point inside the disc from the labeling operation, $\mathrm{I}(j)$ is the intensity at pixel location $j, \sigma_{d}$ is some pixel neighborhood of the location $d, \mu_{\mathrm{I}}$ is the expected intensity levels of the herniated discs, $\sigma_{\mathrm{I}}^{2}$ is the variance of the intensity levels of the herniated discs. Both $\mu_{\mathrm{I}}$ and $\sigma_{\mathrm{I}}^{2}$ are learned from the labeled training data.

On the other hand, the shape potential $U_{\mathrm{S}}$ is defined by the shape features resulting from the ASM. Upon examining the shapes of both the herniated and normal discs, we find that the points $\left[\mathbf{s}_{1}-\mathbf{s}_{8}\right]$ always refer to the shape of the disc while the points $\left[\mathbf{s}_{9}-\mathbf{s}_{11}\right]$ help maintaining the alignment of the disc with the spine. Thus we pick two potentials for defining $U_{\mathrm{S}}$ as follows: 1) $U_{\mathrm{S} 1}$ models the Euclidean distance $e_{1}$ between point 2 ( $\left.\mathrm{s}_{2}\right)$ and point $8\left(\mathbf{s}_{8}\right)$ as labeled in Fig. 4. 2) $U_{\mathrm{S} 2}$ models the sum $e_{2}$ of the major and minor axes of the elliptic disc shape. Both the linear nature of the ASM and the empirical analysis led us to choose Gaussian models for both $U_{\mathrm{S} 1}$ and $U_{\mathrm{S} 2}$. Thus, we define the shape potential by:

$$
U_{\mathrm{S}}(\mathrm{S})=\alpha_{1} U_{\mathrm{S} 1}+\alpha_{2} U_{\mathrm{S} 2}
$$

where $\alpha_{1}$ and $\alpha_{2}$ are tuning parameters, $U_{\mathrm{S} 1}$ and $U_{\mathrm{S} 2}$ are the two potentials for modeling $e_{1}$ and $e_{2}$, respectively. Thus, we define

$$
U_{\mathrm{S} 1}=\frac{\left(e_{1}-\mu_{e_{1}}\right)^{2}}{2 \sigma_{e_{1}}^{2}}
$$

where $e_{1}=\left|\mathbf{s}_{2}-\mathbf{s}_{8}\right|_{2}$ where $\mathbf{s}_{2}$ and $\mathbf{s}_{8}$ are the location coordinates of points 2 and 8 as shown in Fig. $4, \mu_{e_{1}}$ is the expected Euclidean distance between the points $\mathbf{s}_{2}$ and $\mathbf{s}_{8}$, and $\sigma_{e_{1}}^{2}$ is the variance of the Euclidean distances between the points $\mathbf{s}_{2}$ and $\mathbf{s}_{8}$. We learn both $\mu_{e_{1}}$ and $\sigma_{e_{1}}^{2}$ from the training data.

$$
U_{\mathrm{S} 2}=\frac{\left(e_{2}-\mu_{e_{2}}\right)^{2}}{2 \sigma_{e_{2}}^{2}}
$$

where $e_{2}=\left|\mathbf{s}_{1}-\mathbf{s}_{5}\right|_{2}+\left|\mathbf{s}_{3}-\mathbf{s}_{7}\right|_{2}$ where $\mathbf{s}_{1}, \mathbf{s}_{3}, \mathbf{s}_{5}$, and $\mathbf{s}_{7}$ are the location coordinates of points $1,3,5$, and 7, respectively, as shown in Fig. $4, \mu_{e_{2}}$ is the expected sum of the major and minor axes of the disc, $\sigma_{e_{1}}^{2}$ is the variance of the sums of the major and minor axes of the disc. We learn both $\mu_{e_{2}}$ and $\sigma_{e_{2}}^{2}$ from the training data.

\section{EXPERIMENTAL RESULTS}

We validate our proposed model with 33 clinical MRI cases. Each case contains two co-registered volumes: T1- and T2-weighted. For training our model and learn the parameters, we pick the slide where the herniation is present. We then annotate the ground truth by marking the herniated discs. We based this annotation on actual clinical reports from our collaborating radiologist. We consider these reports as our gold standard. We emphasize that inter-observer errors exist in lumbar diagnosis similar to most diagnosis tasks from various imaging modalities. However, MRI shows high inter-observer reliability compared to plain x-ray radiographs 
Table 1. Table shows the results of the cross validation experiment with an average detection accuracy of $91.7 \%$.

\begin{tabular}{|c|ccc|ccc|c|}
\hline Set & E6 & E5 & E4 & E3 & E2 & E1 & Accuracy \\
\hline 1 & 11 & 12 & 10 & 13 & 13 & 13 & $92.3 \%$ \\
2 & 11 & 13 & 13 & 11 & 12 & 10 & $89.7 \%$ \\
3 & 13 & 12 & 11 & 12 & 13 & 13 & $94.9 \%$ \\
4 & 10 & 13 & 13 & 10 & 13 & 11 & $89.7 \%$ \\
5 & 12 & 12 & 13 & 13 & 12 & 9 & $91.0 \%$ \\
\hline 6 & 12 & 10 & 13 & 11 & 11 & 13 & $89.7 \%$ \\
7 & 13 & 11 & 12 & 11 & 13 & 12 & $92.3 \%$ \\
8 & 12 & 9 & 13 & 13 & 13 & 13 & $93.6 \%$ \\
9 & 13 & 11 & 12 & 13 & 10 & 13 & $92.3 \%$ \\
10 & 10 & 13 & 11 & 13 & 13 & 11 & $91.0 \%$ \\
\hline$(\%)$ & 90.0 & 89.2 & 93.1 & 92.3 & 94.6 & 90.8 & - \\
\hline \multicolumn{7}{|c|}{ Average Accuracy } \\
\hline
\end{tabular}

in lumbar area diagnosis, which indicates higher agreement between radiologists when diagnosing MRI than x-rays radiographs. For example, Mulconrey et al. ${ }^{26}$ showed that abnormality detection for degenerative disc and spondylolisthesis with MRI has $\kappa=0.773$ and $\kappa=0.728$, respectively, which is considered high in showing inter-observer reliability where this reliability is considered perfect when $0.8 \leq \kappa \leq 1$.

We perform a cross-validation experiment using the 33 cases to train and test our proposed method. In each round, we separate 13 cases and train on the remaining 20 cases. We perform 10 rounds and each time the cases are selected randomly. We define:

$$
\text { Accuracy }_{i}=\left(1-\frac{1}{K} \sum_{j=1}^{K}\left|g_{i j}-n_{i j}\right|\right) * 100 \%
$$

where Accuracy $_{i}$ represents the classification accuracy (herniated disc detection) at the lumbar disc level $i$ where $1 \leq i \leq 6, K$ is the testing set size in each round (13 cases), $g_{i j}$ is the ground truth binary assignment for disc $i$ in case $j$, and $n_{i j}$ is the resulting binary assignment for disc $i$ from the inference on our model. The binary variables $g_{i}$ and $n_{i}$ are assigned the binary values such that they are 0 if $i$ is a normal disc and 1 if it is a herniated disc.

Table 1 shows the classification results from the cross validation experiment. We achieve an average of over $91 \%$ accuracy on classification of discs as herniated or normal. The table also shows the accuracy at each lumbar level (column) in each cross validation round (row).

The lower two levels (E5: L4-L5) and (E6: L5-S) have the most variability in the lumbar area which misleads the ASM and thus the error increases. However, the method is able to classify them with $90 \%$ accuracy which is promising. The top level (E1: T12-L1) disc is usually small compared to the rest of the lumbar discs which produces similar shape feature values for herniated collapsed discs. We may avoid this by having a separate model for this disc as its shape is different. However, the method achieves over $90 \%$ accuracy which is promising as well. The middle levels (E2: L1-L2), (E3: L2-L3), and (E4: L3-L4) achieve higher classification accuracy as the discs are stable in these levels.

Table 2. Calculation of specificity (91\%) of and sensitivity (94\%).

\begin{tabular}{|c|c|c|c|}
\cline { 3 - 4 } \multicolumn{2}{c|}{} & \multicolumn{2}{c|}{ Gold standard } \\
\cline { 3 - 4 } \multicolumn{2}{c|}{} & herniated & normal \\
\hline \multirow{\vec{\sigma}}{*}{} & herniated & $185(\mathrm{TP})$ & $54(\mathrm{FP})$ \\
$\stackrel{0}{0}$ & & & \\
$\sim$ & normal & $11(\mathrm{FN})$ & $530(\mathrm{TN})$ \\
\hline
\end{tabular}




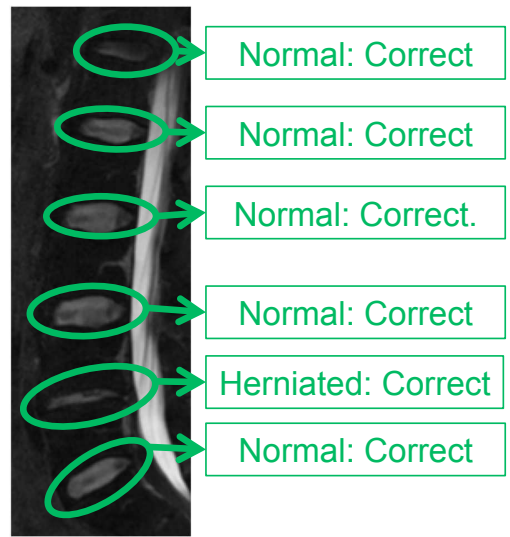

(a)

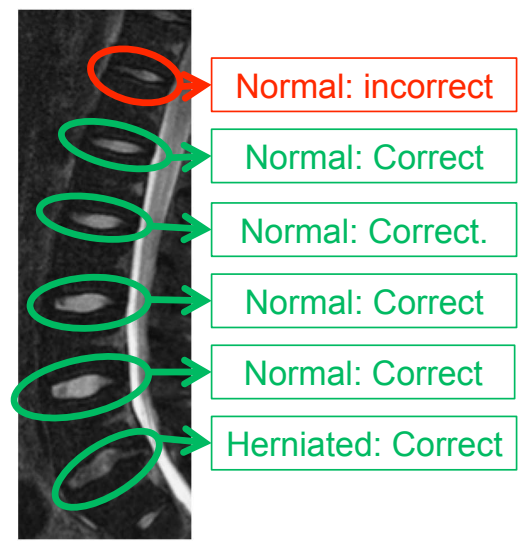

(b)

Figure 7. (a) Level L4-L5 is herniated. All discs are correclty classified. (b) False positive at level T12-L1.

In this dataset, there are 44 herniated discs and the remaining 154 are normal. On average, we notice that clinical cases contain one or two herniated discs, very rare cases we find more. In table 1, each row represents a test on 13 cases $(13 \times 6=78$ discs $)$ which gives a total of 780 discs. Among these 780 discs, we count 196 herniated discs. The total misclassified discs, from the table is, 65 . There are 11 misclassified herniated discs and the remaining 54 misclassified discs are normal. This indicates that our specificity is around $91 \%$ while our sensitivity is around $94 \%$. Table 2 shows the counts of false positives (FP), true positives (TP), false negatives $(\mathrm{FN})$, and true negatives $(\mathrm{TN})$ where:

$$
\begin{aligned}
& \text { Specificity }=\frac{T N}{T N+F P} \\
& \text { Sensitivity }=\frac{T P}{T P+F N}
\end{aligned}
$$

Fig. 7(a) shows an example of a correctly classified case while Fig. 7(b) shows a case with a false positive disc at the top level (T12-L1). In the later case, the disc is very narrow compared to other discs which causes the shape features to mis-classify it as herniated similar to the herniated disc at level (L4-L5) in Fig. 7(a). Most discs at this level are smaller than the rest of the lumbar discs which makes them appear as if the discs collapsed as a result of hernation.

\section{CONCLUSION}

We proposed a probabilistic model for automatic herniation detection that incorporates appearance and shape features of the lumbar intervertebral discs. We presented an application for the use of ASM to extract suitable features for herniation shape detection. We validated our model using 33 abnormal clinical MRI cases. Each case contains at least one herniated disc. We consider the clinical report of the radiologist as our gold standard for herniation condition of each disc. We perform a cross-validation experiment on the 33 cases by leaving 13 cases for testing each round. The overall herniation detection accuracy is around $90 \%$. We also reported specificity of $91 \%$ and sensitivity of $94 \%$.

\section{ACKNOWLEDGEMENT}

This work was supported in part by the New York State Foundation for Science, Technology and Innovation (NYSTAR). 


\section{REFERENCES}

[1] NINDS, "National institute of neurological disorders and stroke (ninds): Low back pain fact sheet," NIND brochure (2008).

[2] Luoma, K., Riihimki, H., and et al., "Low back pain in relation to lumbar disc degeneration," Spine 25, 487-92 (Feb 2000).

[3] Snell, R. S., [Clinical Anatomy by Regions], Lipp. Will. and Wilkins, 8th ed. (2007).

[4] An, H., Anderson, P., and et al, "Disc degeneration," Spine 29, 2677-2678 (Dec 2004).

[5] Kiraly, A. P., Laks, S., Macari, M., Geiger, B., Bogoni, L., and Novak, C. L., "A fast method for colon polyp detection in high-resolution ct data," Int. Congress Series 1268, 983-988 (2004).

[6] iCAD, "Vera look from icad." Computer Aided Diagnosis Tool (www.icadmed.com/veralook.htm) (2009).

[7] White, C. S., Pugatch, R., Koonce, T., Rust, S. W., and Dharaiya, E., "Lung nodule cad software as a second reader: A multicenter study," Academic Radiology 15(3), 326-333 (2008).

[8] iCAD, "Second look digital from icad." Computer Aided Diagnosis Tool (www.icadmed.com/SecondLookDigital.htm) (2009).

[9] iCAD, "Spectra look digital from icad." Computer Aided Diagnosis (www.icadmed.com/breastmri.htm) (2009).

[10] iCAD, "Vivid look from icad." Computer Aided Diagnosis (www.icadmed.com/prostatemri.htm) (2009).

[11] Zhou, Z., Liua, B. J., , and Lea, A. H., "Cadpacs integration tool kit based on dicom secondary capture, structured report and ihe workflow profiles," Computerized Medical Imaging and Graphics 31, 346-352 (June 2007).

[12] Domino, D., "Integration of cad with pacs breaks down barrier to its use," Diagnostic Imaging , 25-29 (2009).

[13] Corso, J. J., Alomari, R. S., and Chaudhary, V., "Lumbar disc localization and labeling with a probabilistic model on both pixel and object features.," in [Proc. of MICCAI], LNCS Part 1 5241, 202-210, Springer (2008).

[14] Alomari, R. S., Corso, J. J., and Chaudhary, V., "Abnormality detection in lumbar discs from clinical mr images with a probabilistic model," in [Proc. of CARS], (2009).

[15] Alomari, R. S., Corso, J. J., Chaudhary, V., and Dhillon, G., "Desiccation diagnosis in lumbar discs from clinical mri with a probabilistic model," in [Proc. of ISBI'09], 546-549 (2009).

[16] Alomari, R. S., Corso, J. J., Chaudhary, V., and Dhillon, G., "Computer-aided diagnosis of lumbar disc pathology from clinical lower spine mri," International Journal of Computer Assisted Radiology and Surgery. (2009). in press.

[17] Cootes, T. F. and Taylor, C. J., "Statistical models of appearance for medical image analysis and computer vision," in [Proc. of SPIE Medical Imaging], (2001).

[18] Swarm, "Interactive incorporation (viewmedica) - patient educatuion system.," (2007).

[19] Bounds, D. G., Lloyd, P., and et al, "A multilayer perceptron network for the diagnosis of low back pain," 2, 481-489 (July 1988).

[20] Vaughn, M., "Using an artificial neural network to assist orthopaedic surgeons in the diagnosis of low back pain," (2000).

[21] Tsai, M. and et al, "A new method for lumbar herniated inter-vertebral disc diagnosis based on image analysis of transverse sections," CMIG 26(6), 369-380 (2002).

[22] Roberts, M., Cootes, T., and et al, "Quant. vertebral fracture detection on dxa images using shape and appearance models," Acad. Radiology 14, 1166-1178 (Oct 2007).

[23] Videman, T., Batti, M., and et al, "Determ. of the progression in lumbar degeneration: a 5-year follow-up study of adult male monozygotic twins," Spine 31, 671-678 (2006).

[24] Niemelinen, R., Videman, T., and et al, "Quantitative measurement of intervertebral disc signal using mri," Clin. Rad. 63(3), $252-255$ (2008).

[25] Cootes, T. F., Taylor, C. J., Cooper, D. H., and Graham, J., "Active shape models - their training and application," Comput. Vis. Image Underst. 61(1), 38-59 (1995).

[26] Mulconrey, D. and Knight, R., "Interobserver reliability in the interpretation of diagnostic lumbar mri and nuclear imaging," Spine 6, 177 - 184 (2006). 